\title{
A Novel Cascade Benzyne Nucleophilic Addition/Fries Rearrange- ment for Entry into 2,3-Disubstituted Phenols
}

Sarah M. Bronner*

Daniel Lee

Vlad Bacauanu

Patrick Cyr

Synlett 2017, 28, 799.

In the e-first version of this article, compound $\mathbf{1}$ in Table 2 was missing a triflate group. This has been corrected for the final online and print versions. 